\title{
Correction to: The Association Between Renal Tubular Dysfunction and Zinc Level in a Chinese Population Environmentally Exposed to Cadmium
}

\author{
Xiao Chen ${ }^{1,2} \cdot$ Zhongqiu Wang $^{2} \cdot$ Guoying Zhu ${ }^{3} \cdot$ Gunnar F. Nordberg ${ }^{4} \cdot$ Xiaoqiang Ding $^{1} \cdot$ Taiyi Jin $^{5}$ \\ Published online: 26 November 2020 \\ (C) Springer Science+Business Media, LLC, part of Springer Nature 2020
}

Correction to: Biological Trace Element Research https://doi.org/10.1007/s12011-018-1304-3

The original version of this article unfortunately contained a mistake. The authors have found that there are several unintended mistakes in Table 3. The correct Table 3 is presented here. The overall conclusions are not altered. The authors apologise for any inconvenience this may have caused.

Publisher's Note Springer Nature remains neutral with regard to jurisdictional claims in published maps and institutional affiliations.

Table 3 Odds ratios (ORs) and 95\% confidence intervals (CIs) of renal dysfunction and UCd, BCd, UCa, Zn/Cd ratio, SZn, HZn

\begin{tabular}{|c|c|c|c|c|}
\hline & \multirow[b]{2}{*}{$\mathrm{n}$} & \multicolumn{3}{|l|}{ Odds ratio $(95 \% \mathrm{CI})$} \\
\hline & & & Model 1 & Model 2 \\
\hline \multirow[t]{4}{*}{$\mathrm{UCd}(\mu \mathrm{g} / \mathrm{g}$ cr $)$} & 84 & Reference $(<3.6)$ & 1 & 1 \\
\hline & 82 & $3.6-8.7$ & $1.32(0.56-3.16)$ & $1.29(0.71-2.4)$ \\
\hline & 81 & $8.7-16.9$ & $2.83(1.26-6.36)$ & $2.29(1.01-5.25)$ \\
\hline & 84 & $\geq 16.9$ & $4.29(1.93-9.56)$ & $2.40(1.04-5.68)$ \\
\hline \multirow[t]{4}{*}{$\mathrm{BCd}(\mu \mathrm{g} / \mathrm{L})$} & 83 & $\overline{\text { Reference }}(<1.85)$ & 1 & 1 \\
\hline & 83 & $1.85-7.34$ & $1.46(0.51-4.14)$ & $1.43(0.50-4.08)$ \\
\hline & 83 & $7.34-14.89$ & $6.30(2.51-16.11)$ & $5.72(2.24-14.59)$ \\
\hline & 82 & $\geq 14.89$ & $10.42(4.14-26.21)$ & $10.3(4.07-26.04)$ \\
\hline \multirow[t]{4}{*}{$\mathrm{UCa}(\mathrm{g} / \mathrm{g}$ cr) } & 78 & $\overline{\text { Reference }}(<0.10)$ & 1 & 1 \\
\hline & 83 & $0.10-0.17$ & $1.87(0.80-4 . .36)$ & $2.16(0.85-5.50)$ \\
\hline & 88 & $0.17-0.28$ & $2.02(0.84-4.84)$ & $2.49(1.02-6.45)$ \\
\hline & 82 & $\geq 0.28$ & $5.40(2.34-12.45)$ & $5.42(2.11-13.90)$ \\
\hline \multirow[t]{4}{*}{$\mathrm{Zn} / \mathrm{Cd}$} & 83 & $\overline{\text { Reference }}(<100.0)$ & 1 & 1 \\
\hline & 88 & $100.0-200.0$ & $0.47(0.24-0.89)$ & $0.40(0.19-0.84)$ \\
\hline & 81 & $200.0-600.0$ & $0.16(0.07-0.36)$ & $0.14(0.06-0.37)$ \\
\hline & 79 & $\geq 600.0$ & $0.06(0.02-0.16)$ & $0.06(0.02-0.18)$ \\
\hline \multirow{3}{*}{$\mathrm{SZn}(\mathrm{mg} / \mathrm{L})$} & 111 & $\bar{R}$ eference $(<1.14)$ & 1 & 1 \\
\hline & 171 & $1.14-1.62$ & $0.66(0.39-1.14)$ & $0.58(0.31-1.08)$ \\
\hline & 49 & $\geq 1.62$ & $0.38(0.12-1.23)$ & $0.26(0.07-0.99)$ \\
\hline \multirow[t]{3}{*}{$\mathrm{HZn}(\mathrm{mg} / \mathrm{g})$} & 42 & $\bar{R}$ eference $(<0.12)$ & 1 & \\
\hline & 48 & $0.12-0.14$ & $0.57(0.18-1.83)$ & $0.59(0.17-2.02)$ \\
\hline & 58 & $\geq 0.14$ & $0.12(0.03-0.49)$ & $0.09(0.02-0.48)$ \\
\hline
\end{tabular}

The online version of the original article can be found at https://doi.org/10. 1007/s12011-018-1304-3

Xiaoqiang Ding

ding_xiaoqiang@zs-hospital.sh.cn

$\triangle$ Taiyi Jin

071114298@fudan.edu.cn

1 Department of Nephrology, Shanghai Key Laboratory of Kidney and Dialysis, Zhongshan Hospital Fudan University, 180 Fenglin road, Shanghai 200032, China
2 Department of Radiology, Affiliated Hospital of Nanjing University of Chinese Medicine, Nanjing 210029, China

3 Institute of Radiation Medicine, Fudan University, 2094 Xietu road, Shanghai 200032, China

4 Department of Public Health and Clinical Medicine, Umeå University, 90187 Umeå, Sweden

5 Department of Occupational Medicine, School of Public Health, Fudan University, 150 Dongan road, Shanghai 200032, China 